\title{
EVOLUÇÃO DO COMANDO NUMÉRICO COMPUTADORIZADO
}

\author{
EVOLUTION OF THE COMPUTERIZED NUMERICAL COMMAND
}

\author{
Alex Júnior Orlando - alexousadia@live.com \\ Faculdade de Tecnologia de Taquaritinga - Taquaritinga - São Paulo - Brasil \\ Ramilio Reis Filho - ramilio.ramalho@hotmail.com \\ Faculdade de Tecnologia de Taquaritinga - Taquaritinga - São Paulo - Brasil
}

DOI: 10.31510/infa.v18i1.1153

Data de submissão: 17/04/2021

Data do aceite: 09/07/2021

Data da publicação: 30/07/2021

\begin{abstract}
RESUMO
$\mathrm{O}$ advento da internet trouxe consigo o surgimento de avançadas tecnologias as quais atingiram de forma significativa vários setores da sociedade, em especial as indústrias. Como forma de ganhar um mercado cada vez mais competitivo, as indústrias tem buscado investir em modernos equipamentos, que usam avançadas tecnologias. O Comando Numérico Computadorizado (CNC) é uma das tecnologias utilizadas pelas indústrias, e que tem como objetivo promover um novo padrão de produção, realizado com mais segurança, qualidade do produto, agilidade e maior rendimento. $\mathrm{O}$ objetivo deste estudo é evidenciar a evolução e importância do CNC para as organizações. A pesquisa é uma Revisão de Literatura com consultas em artigos, livros e documento dispostos em simpósios e congressos. A literatura estudada traz a importância e a evolução constante que tem acontecido com o CNC, uma vez que sua evolução se faz necessário, especialmente frente a uma sociedade globalizada e inserida na era digital, onde as tecnologias se transformam e evoluem de forma impactante.
\end{abstract}

Palavras-chave: Comando Numérico Computadorizado. Evolução. Tecnologia.

\begin{abstract}
The advent of the internet has brought with it the emergence of advanced technologies which have significantly affected various sectors of society, especially industries. As a way of gaining an increasingly competitive market, industries have sought to invest in modern equipment, which uses advanced technologies. The Computer Numerical Command (CNC) is one of the technologies used by the industries, which aims to promote a new production standard, carried out with more safety, product quality, agility and higher yield. The purpose of this study is to highlight the evolution and importance of CNC for organizations. The research is a Literature Review with consultations on articles, books and documents arranged in symposia and congresses. The studied literature brings the importance and constant
\end{abstract}


evolution that has happened with the $\mathrm{CNC}$, since its evolution is necessary, especially in the face of a globalized society and inserted in the digital age, where technologies are transformed and evolve in an impactful way.

Keywords: Computer Numerical Command. Evolution. Technology.

\section{INTRODUÇÃO}

As evoluções tecnológicas trouxeram profundas mudanças em todo o contexto social e se estenderam para os modelos de produção realizados nas indústrias, as quais têm inserido em seu processo produtivo tais tecnologias como forma de alavancar a produção (ARBIX et al., 2017).

O século XX viu nascer á era da informática e o mundo então mudou o significativo avanço da tecnologia propiciou ás indústrias, num clima de grande concorrência, buscar novos recursos que lhes fornecessem um produto de alta qualidade com um mínimo de custo. Grandes investimentos foram aplicados em modernos equipamentos para ganhar mercado (SILVA, 2002).

A ideia de desenvolver o presente artigo surgiu do interesse no assunto, buscando entender com mais profundidade tudo voltando às máquinas, trajetórias, trabalhadores na indústria e realidade virtual.

Ainda hoje há necessidade de estar atento aos recursos que surgem, pois a informática é uma ferramenta que não estaciona no tempo, possibilitando avanços progressivos em tecnologia, como podemos observar com o advento do $\mathrm{CNC}$ o qual une-se à metalmecânica, impondo às indústrias um novo padrão de produção assentada em 100\% de rendimento, alta qualidade do produto, agilidade, segurança e confiabilidade, o que torna as máquinas convencionais obsoletas, além de exigir do profissional a busca desta nova tecnologia (SILVA, 2002).

Diante do fato de que as empresas estão buscando cada vez mais cada vez mais aperfeiçoar sem recursos tecnológicos e processos, a ideia é entender como o CNC auxilia na busca por esses resultados através de mercado cada vez mais competitivo com produtos novos e ainda com grande escala de fabricação.

O CNC traz segmentos e linhas de raciocínios muito alinhadas do público-alvo ele se revoluciona conforme os produtos ligados a ela se revoluciona, a importância deste justifica a realização do presente estudo. 


\section{WWEBPARESTEENOLLGGCA}

Dentro deste contexto o objetivo deste estudo é evidenciar a evolução do CNC e como este fator se faz importante nas indústrias como crescimento e melhora dos resultados da produção.

Para este estudo foi realizado levantamento bibliográfico através de pesquisas em revistas, artigos e livros, que tem como foco o processo de surgimento e evolução do CNC.

\section{O SURGIMENTO E EVOLUÇÃO DO CNC}

O principal fator desencadeante para que os meios industriais buscassem evoluir ainda mais, foi a Segunda Guerra Mundial. Nesse período as necessidades de mais aviões, tanques, barcos, navios, armas e outros trouxe a demanda por uma produção de larga escala e com maior precisão. Esse era o momento certo para que fossem criadas máquinas automáticas, capazes de produzir em grande escala, com grande precisão, e que fossem independentes da mão-de-obra humana (CASSANIGA, 2005).

Em 1800 o francês Joseph Marie Jacquard, criou um tear mecânico que dispunha de uma leitora automática de cartões, era a primeira maquina que utilizava o Comando Numérico, e funcionaria com instruções em números que eram controlados automaticamente (BRAGAGNOLO, 2018).

A figura 1 traz a foto da máquina e tear.

\section{Figura 1: Máquina de tear de Jacquard que funcionava por Comando Numérico}

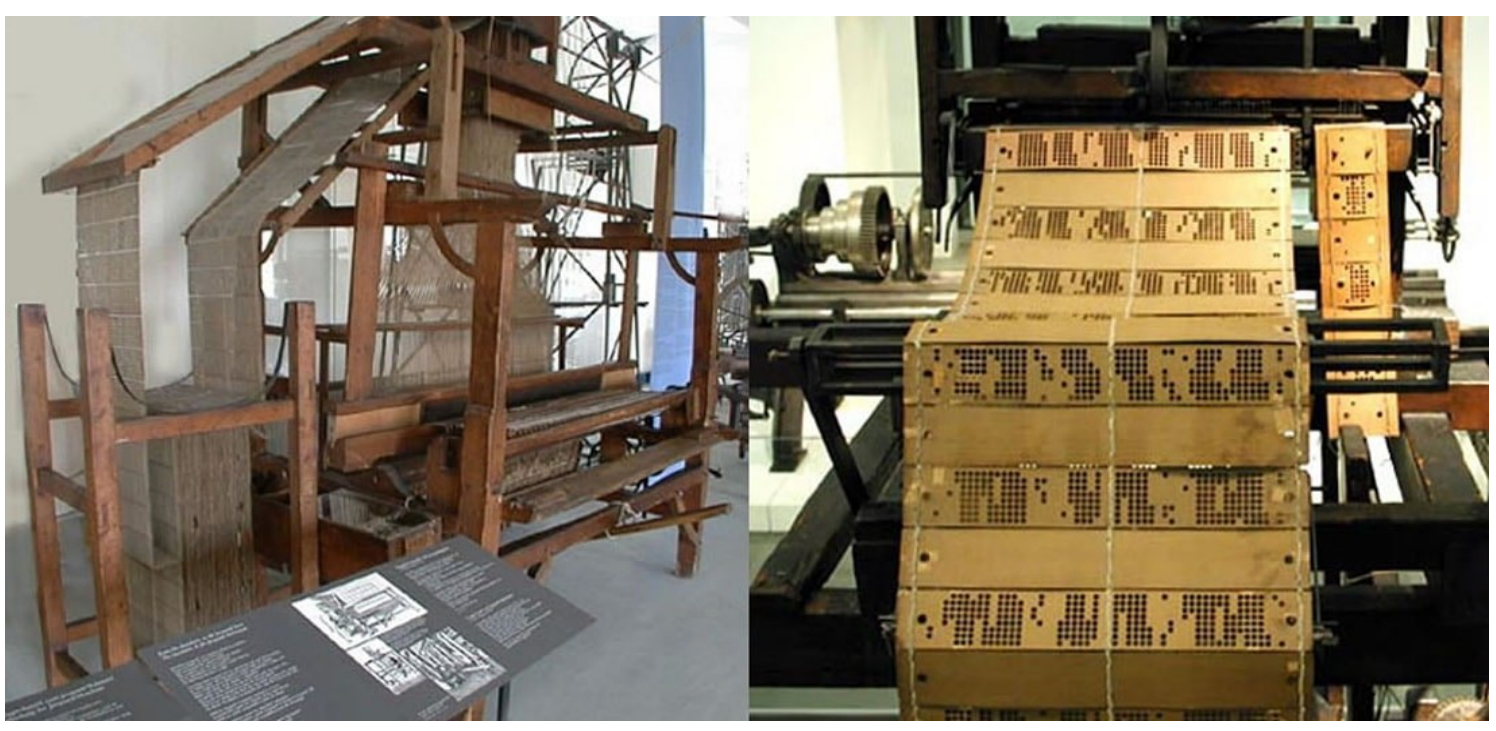

Fonte: Cunha (2017) 


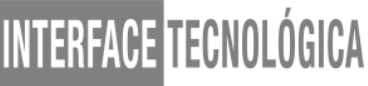

Hermam Hollerith em 1900 criou um sistema de armazenamento de dados, por meio de cartões perfurados os quais seriam utilizados para o sistema de recenseamento dos EUA. Em 1912 Scheyer pediu a patente da máquina de cortar algodão, a qual controlava os movimentos por meio de uma gravação prévia feita em uma folha perfurada de papel (SOUZA, 2012).

A figura 2 traz a foto da máquina de cartão perfurado de Herman Hollerith.

\section{Figura 2: Máquina de cartão perfurado de Hollerith}

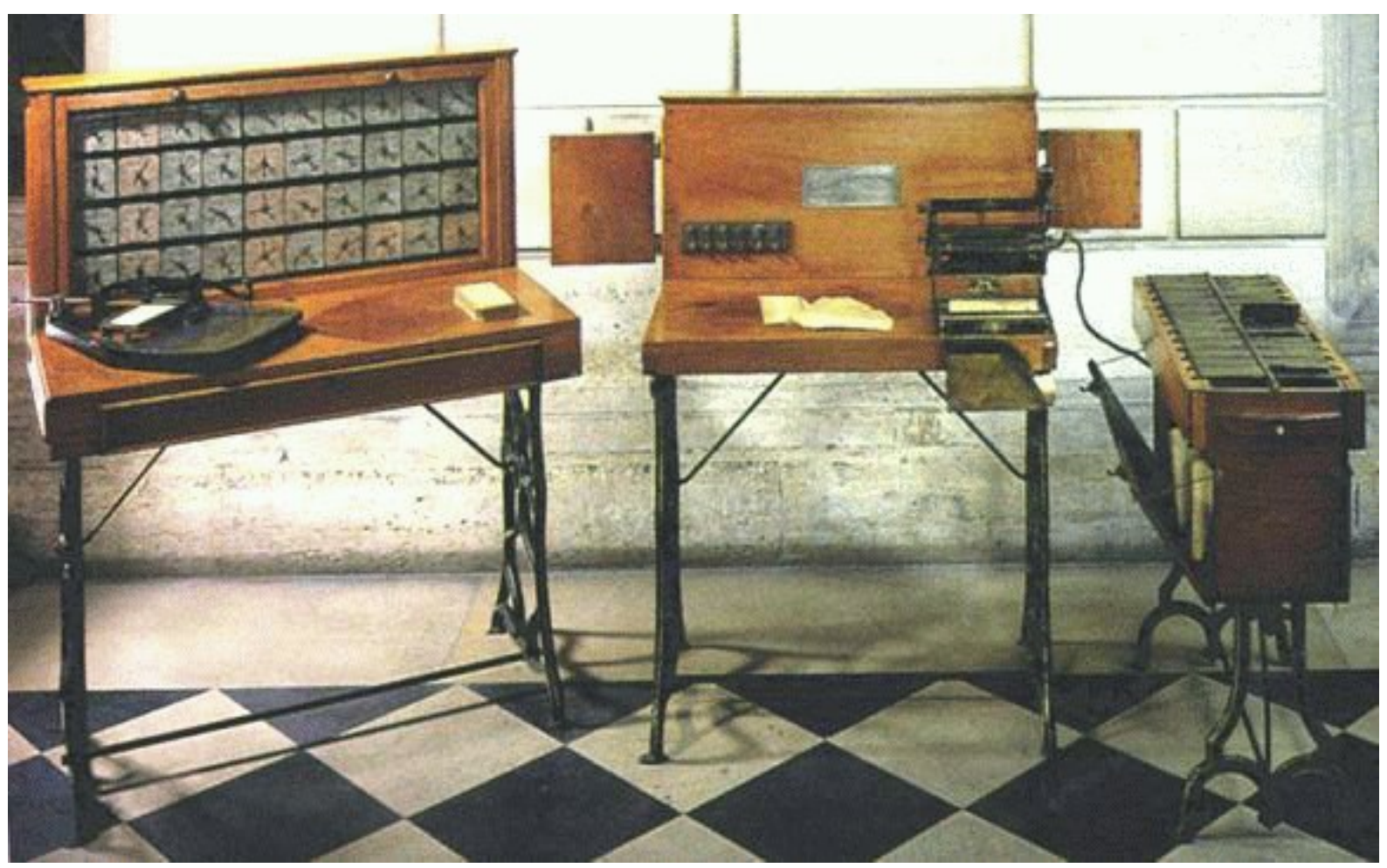

Fonte: Mundo CNC (2020)

As máquinas que utilizavam cartões perfurados tinham números em códigos, os quais eram gravados em fitas magnéticas, sendo o mais comum conhecido o disquete ou eram gravadas em um banco de dados centralizados (AZEVEDO, 2020).

Segundo Souza (2012) o primeiro protótipo de máquina com Controle Numérico foi feito em 1952 em Massachusetts Institute of Technology, a qual tratava de uma fresadora vertical copiadora, retrabalhada via "retrofitting" com servomotores, utilizada no fresamento frontal de alumínio. Todos os dados de entrada obtidos eram fornecidos por uma fita perfurada. Os testes apresentaram sucesso, onde as peças foram fabricadas de forma precisa e repetitiva, sem que houvesse intervenção do operador. 


\section{WWEBPARESTEENOLLGGCA}

A fresadora criada possuía três eixos, a Hydrotel, da Cincinnati Milling Machine Company, como alvo das novas experiências. O autor descreve que os controles e comandos convencionais foram substituídos pelo Comando Numérico. $\mathrm{O}$ autor afirma que a criação de máquinas que utilizavam o $\mathrm{CN}$ como a Força Aérea Norte Americana começaram a ter grande desenvolvimento, uma vez que as peças complexas e de grande precisão que eram utilizadas para a fabricação de aeronaves, começaram a ser produzida de forma simples e rápida (CASSANIGA, 2005).

Um sistema de controle numérico tem a finalidade de controlar as ações de uma ou mais máquinas as quais realizam a interpretação automática que estão expressas em números. Os dados são convertidos como distâncias, ângulos temperaturas e outros. As máquinas que são numericamente controladas recebem informações digitais (AZEVEDO, 2020).

A tecnologia do Controle Numérico Computadorizado (CNC) surgiu segundo a experiência da Parsons Corporation, a qual era uma pequena empresa que fabricava hélices e rotores de helicópteros. Em 1946, realizaram a aplicação do conceito de controle por números em uma máquina de usinagem convencional conectada a um computador alimentado por informações de cartões perfurados (MCNC, 2018).

Antes de entender todo o processo de evolução do CNC é importante definir o conceito desse comando. Sendo assim, o CNC é a evolução do $\mathrm{CN}$, e que veio substituir o controle por hardware pelo controle por meio do software (SOUZA, 2012).

A maior diferença entre máquinas que usam o $\mathrm{CN}$ e as que usam $\mathrm{CNC}$, é que as primeiras não precisam de memória, apenas leem os comandos e os executam sem muitas alterações. Já as CNC são providas de memória e realizam alterações (MCNC, 2018).

O CNC é um sistema onde um computador é utilizado para fazer o controle da máquina. O programa utilizado para o controle pode ser feito em um sistema integrados de projeto (CAD-D) e fabricação (CAM, CAPP) os quais também podem ser usados para que sejam vistos os possíveis problemas especialmente no processo real de usinagem. O programa também pode ser feito na máquina que possui tela (SOUZA, 2012).

De acordo com Cassaniga (2005) foram criados dois tipos de sistemas para o controle numérico: o Controle Numérico Direto (CND) e o Controle Numérico Computadorizado (CNC). A diferença entre os dois sistemas é que no CND um computador central controla várias máquinas e no $\mathrm{CNC}$ cada máquina tem seu próprio processador, algo de forma individual. 


\section{WTEREFAGE TECNOLOOGGCA}

Diante dessa realidade a indústria de máquinas ferramenta passou a fabricar e vender máquinas com Controle Numérico. Passado um tempo essas máquinas forma equipadas com CNC, o que aumentou a flexibilidade, precisão e versatilidade (SOUZA, 2012).

Azevedo (2020) descreve que as operações de manufatura tem utilizado o CNC com muito sucesso, e a cada dia diante de tantas evoluções tecnológicas muitos sistemas tendem a ser alimentados pelo $\mathrm{CNC}$, que podem ir desde maquinas de usinagem até máquinas de costura. Muitas são as máquinas que utilizam o CNC como: maquinas de corte a laser, dobradeiras de tubos, máquinas de impressões, máquinas de montagens eletrônicas, sistemas industriais e outros.

A figura 3 traz os modelos das primeiras máquinas que usaram o Comando Numérico Computadorizado.

\section{Figura 3: Máquina com Comando Numérico Computadorizado}

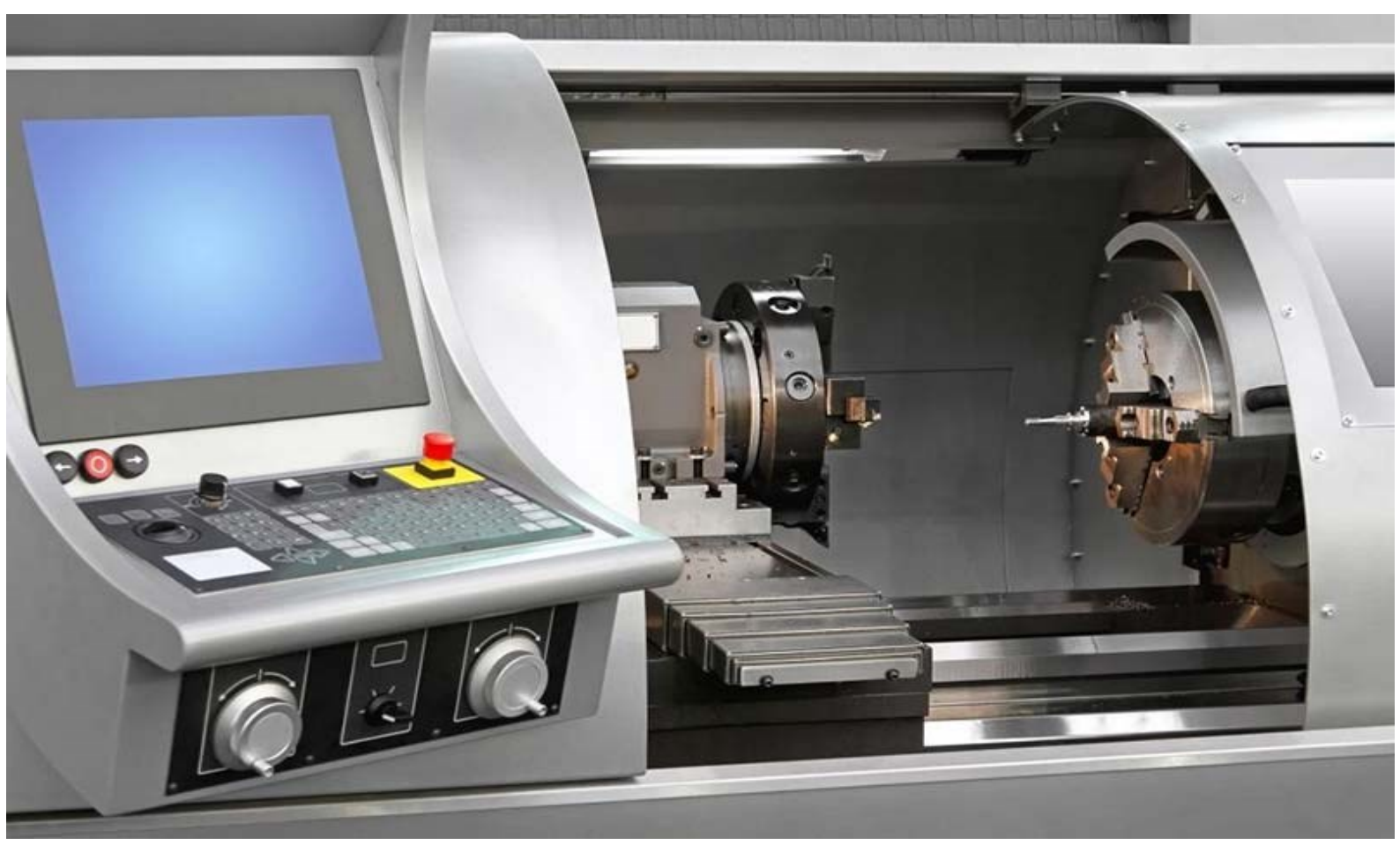

Fonte: M\&S industrial (2019)

Cassaniga (2005) esclarece que com o processo evolutivo, as máquinas que utilizam o sistema de $\mathrm{CNC}$ trouxeram a integração dos sistemas e com isso a redução dos tamanhos físicos dos comandos, além do aumento considerável da capacidade de armazenamento. No Brasil as máquinas controladas por CNC chegaram em 1967 as quais vinham dos Estados 
Unidos. Na década de 70 o Brasil viu surgir as primeiras máquinas CNC (Controle Numérico Computadorizado), e no Brasil surge as primeiras máquinas $\mathrm{CN}$ de fabricação nacional. $\mathrm{O}$ autor esclarece que desde então a evolução é contínuos sendo os CNC envoltos por tecnologias de última geração, melhorando assim a capacidade e confiabilidade desses sistemas tão usados pelas indústrias atuais.

\section{METODOLOGIA}

Segundo Markoni e Lakatos (1999) a pesquisa bibliográfica, ou de fontes secundárias, tem como objetivo englobar toda a bibliografia já publicada em relação ao tema em estudo. Sendo assim a pesquisa bibliográfica inclui publicações em revistas, livros, pesquisas, monografias e teses.

Os dados para esta pesquisa foram realizados entre janeiro e fevereiro de 2021. Para a estrutura deste trabalho foram utilizados artigos científicos, livros e publicações em websites. As bases de dados pesquisadas foram Google Acadêmico e SciELO. Como critério de inclusão utilizou-se a data de publicação (últimos 15 anos) e o idioma (português). Todos os artigos e documentos datados fora do período de publicação e em outro idioma forma excluídos.

Toda a pesquisa realizada possibilitou um maior conhecimento e embasamento sobre o tema foco deste estudo.

Ao total foram encontrados 5 artigos com o tema proposto conforme quadro 1 abaixo.

Quadro 1: Sínteses dos Documentos Encontrados

\begin{tabular}{|c|c|c|c|}
\hline Título & Autor & Ano & Objetivos \\
\hline $\begin{array}{lll}\text { Os } & \text { primórdios } & \text { do } \\
\text { Controle Numérico } & \end{array}$ & AZEVEDO, A. L. de & 2020 & $\begin{array}{l}\text { Evidenciar o conceito } \mathrm{e} \\
\text { como surgiu o processo de } \\
\text { Controle Numérico para as } \\
\text { máquinas }\end{array}$ \\
\hline $\begin{array}{l}\text { O Brasil e a nova onda } \\
\text { de manufatura avançada. }\end{array}$ & $\begin{array}{ll}\text { ARBIX. } & \text { G.; } \\
\text { SALERNO, M. } & \text { S.; } \\
\text { ZANCUL, } & \text { E.; } \\
\text { AMARAL, } & \text { G.; } \\
\text { LINS, L. M. } & \end{array}$ & 2017 & 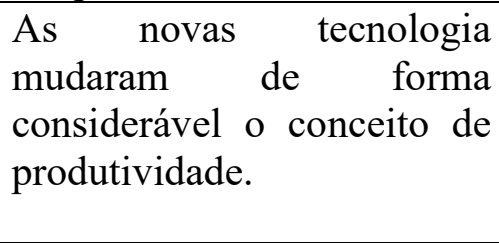 \\
\hline $\begin{array}{l}\text { Fácil Programação do } \\
\text { Controle Numérico } \\
\text { FANUC }\end{array}$ & CASSANIGA, F. A. & 2005 & $\begin{array}{l}\text { Trazer o conceito do } \mathrm{CN} \text {, } \\
\mathrm{CNC} \text { e a evolução desses } \\
\text { comandos até os dias atuais. }\end{array}$ \\
\hline
\end{tabular}




\begin{tabular}{|c|c|c|c|}
\hline $\begin{array}{l}\text { CNC-Programação de } \\
\text { comandos numéricos } \\
\text { computadorizados } \\
\text { torneamento }\end{array}$ & SILVA, S & 2002 & $\begin{array}{l}\text { Evidenciar o conceito do } \\
\text { CNC. }\end{array}$ \\
\hline $\begin{array}{lr}\text { Vantagens } & \mathrm{e} \\
\text { Desvantagens } & \text { do } \\
\text { Controle } & \text { Numérico } \\
\text { Computadorizado } \\
\text { (CNC). }\end{array}$ & SOUZA, C. E. D. de. & 2020 & $\begin{array}{l}\text { A tecnologia trouxe a } \\
\text { evolução das máquinas, } \\
\text { fator importante para o setor } \\
\text { industrial que cresce de } \\
\text { forma considerável. }\end{array}$ \\
\hline
\end{tabular}

Fonte: Elaborado pelo autor segundo os resultados encontrados

\section{RESULTADOS E DISCUSSÃO}

As indústrias têm evoluído de forma considerável e inserido em seu processo produtivo, novas tecnologias, como forma de qualificar e alavancar a produção, conseguindo assim manter-se em um mercado altamente competitivo e exigente, voltado para o uso constante de novas tecnologias.

Tessarini Junior e Saltorato (2018) descrevem que a nova modalidade de indústria ou a Indústria 4.0 tem como característica o uso das potentes e avançadas tecnologias de informação e comunicação no ambiente de produção.

A Indústria 4.0 devido ao uso das novas tecnologias tem como objetivo aumentar a flexibilidade, a velocidade, a produtividade e a qualidade dos processos produtivos (BCG, 2015).

Couto (2019) destaca que aas máquinas que usam o CNC para atender os objetivos da Indústria 4.0 precisam atender os requisitos como: conectividade, flexibilidade, velocidade, sincronismo e inteligência.

Sobre as vantagens e desvantagens do uso do CNC Bezerra (2018) cita como vantagens:

- Boa precisão;

- Alta produtividade;

- Baixos custos;

- Redução do tempo entre fabricação e entrega.

Já sobre as desvantagens Bezerra (2018) cita:

- Custo inicial alto;

- Exigência de manutenção mais especializada, o que pode gerar um maior custo; 


\section{WTEREFAEETEENOLOGGCA}

- Tempo de programação alto.

Oliveira (2016) descreve que as máquinas controladas por CNC existem em todos os lugares, desde as pequena lojas até as grandes indústrias. Em algumas indústrias por exemplo, as indústrias que usam tecnologia avançada, quase ou todas as etapas de produção são realizadas por tais máquinas.

A figura 4 traz o exemplo de máquina mais evoluída existente no mercado que trabalha sob o CNC.

\section{Figura 4: Robô CNC}

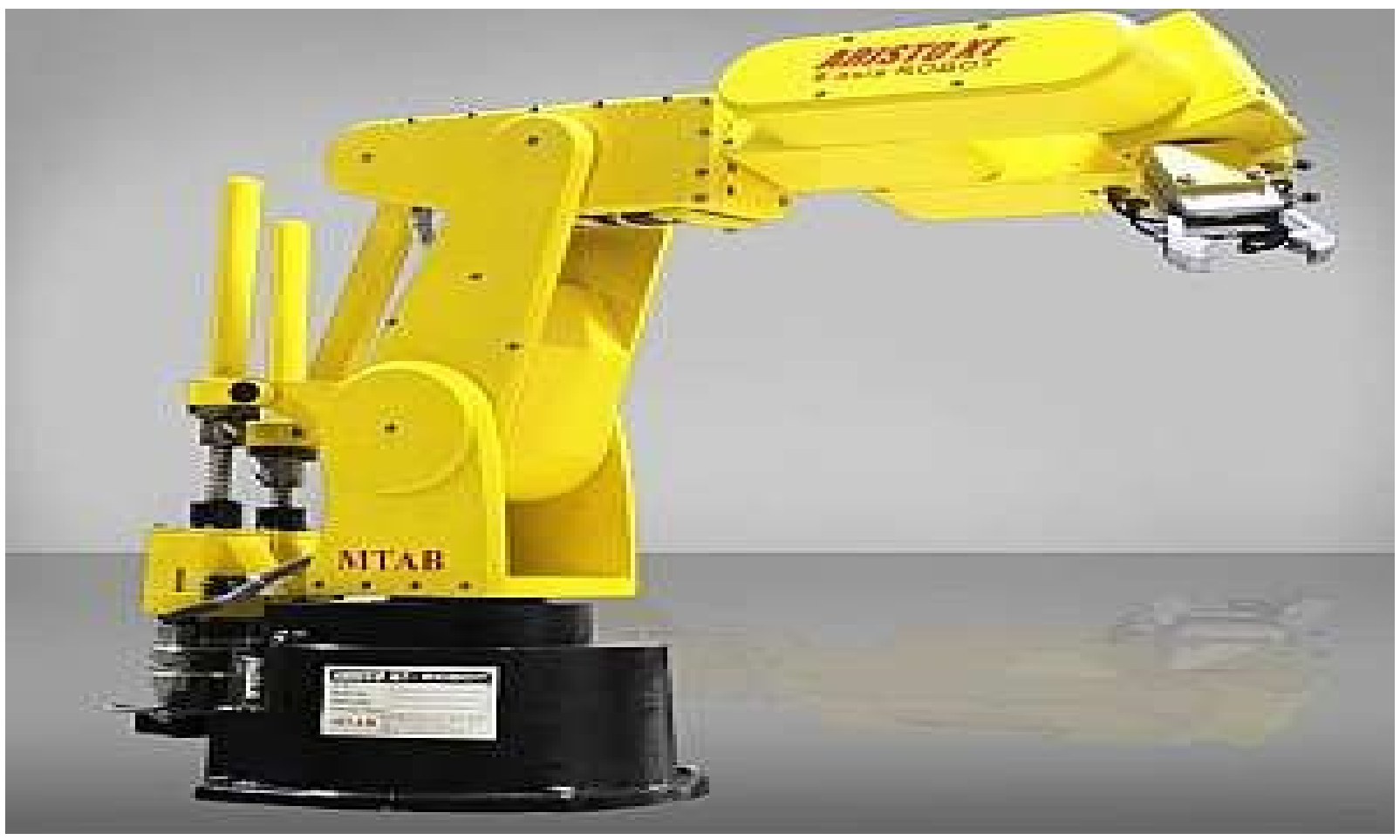

Fonte: Shopenc (2020)

\section{CONCLUSÃO}

As tecnologias evoluíram e passaram a ser inseridas com muita intensidade dentro de todo o mercado de trabalho. Diversos setores da sociedade tem visto a necessidade e importância de desenvolverem seus trabalhos baseados dentro dos conceitos das novas tecnologias. 
$\mathrm{O}$ advento da internet e a evolução do $\mathrm{CNC}$ trouxeram profundas e consideráveis transformações, especialmente para o setor industrial. Desde que surgiram as indústrias buscam investir em tecnologia como forma de qualificar e produzir em grande quantidade.

Todos esses avanços tecnológicos tem contribuído para que o sistema produtivo seja mais eficiente, apresente maior rendimento, segurança nas informações, além de processos mais padronizados.

O uso de computadores para o controle das máquinas é uma realidade que tem sido cada vez mais utilizado dentro das indústrias, um caso notório são as Indústrias 4.0 que buscam inserir em seu processo produtivo as novas tecnologias, dispondo da manufatura avançada, em que computadores e robôs desempenham países antes realizados por humanos.

A eficiência de máquinas com $\mathrm{CNC}$ tem sido evidenciada pelas indústrias que buscam investir em tecnologia. A cada dia o uso de robôs dentro das indústrias tem crescido e mostrado a sua eficiência.

A literatura deixa evidente que a opção por máquinas controladas por $\mathrm{CNC}$ tende a apresentar amplo crescimento, forçando as empresas a investirem nessa área, objetivando sempre o alcance da qualidade, produção em massa e segurança das informações.

\section{REFERÊNCIAS}

ARBIX. G.; SALERNO, M. S.; ZANCUL, E.; AMARAL, G.; LINS, L. M. O Brasil e a nova onda de manufatura avançada. 2017. Disponível em:

https://www.scielo.br/pdf/nec/v36n3/1980-5403-nec-36-03-29.pdf. Acesso em: 10 jan. 2021.

AZEVEDO, A. L. de. Os primórdios do controle numérico. 2020. Disponível em: https://www.mundocnc.com.br/historico/. Acesso em: 12 jan. 2021.

BCG. BOSTON CONSULTING GROUP. Industry 4.0: The future of productivity and growth in manufacturing industries. BCG Perspectives, 2015. Disponível em: https://www.bcgperspectives.com/content/articles/engineered_products_project_business_in dustry_40_future_productivity_growth_manufacturing_industries/. Acesso em: 10 jan. $2 \overline{0} 21$.

BEZZERRA, P. P. L. Implementação de Comando Numérico Computadorizado em uma furadeira de bancada. 2018. Disponível em: http://repositorio.ufc.br/bitstream/riufc/54928/3/2018_tcc_pplbezerra.pdf. Acesso em: 20 jan. 2021. 
BRAGAGNOLO, R. P. Desenvolvimento de uma fresadora didática de comando numérico computadorizado de pequeno porte. 2018. Disponível em: https://bibliodigital.unijui.edu.br:8443/xmlui/bitstream/handle/123456789/5398/Rodrigo\%20 Pires\%20Bragagnolo.pdf?sequence=1\&isAllowed=y. Acesso em: 10 jan. 2021.

CASSANIGA, F. A. Fácil Programação do Controle Numérico - FANUC. Cnc: São Paulo. 2005.

COUTO, S. Z. do. Como deverão ser as máquinas CNC na Indústria 4.0? 2019. Disponível em: https://www.industria40.ind.br/artigo/18705-como-deverao-ser-as-maquinas-cnc-na-industria-40. Acesso em: 10 jan. 2021.

CUNHA, R. O Tear Jacquard não só revolucionou a indústria têxtil mas foi o primeiro computador do mundo. 2017. Disponível em: https://www.stylourbano.com.br/o-tearjacquard-nao-so-revolucionou-a-industria-textil-mas-foi-o-primeiro-computador-do-mundo/. Acesso em: 12 mar. 2021.

MARCONI, M. A.; LAKATOS, E. M. Metodologia do trabalho científico. 4.ed. São Paulo: Atlas, 1999.

MCNC. MANUTEC. A importância do retrofitting de máquinas e equipamentos. 2018. Disponível em: http://www.manutec.ind.br/index.php/blog-manutec/52-aimportancia-doretrofitting-de-maquinas-e-equipamentos Acesso em: 10 jan. 2021.

M\&S Industrial. CNC - Comando Numérico Computadorizado. 2019. Disponível em: https://mesindustrial.com.br/cnc-comando-numerico-computadorizado/. Acesso em: 20 fev. 2021.

OLIVEIRA, L. F. dos S. Desenvolvimento de um robô cartesiano acionado por CNC. Disponível em: https://repositorio.unisc.br/jspui/bitstream/11624/2153/1/Luis\%20Felipe.pdf. Acesso em: 20 jan. 2021.

SILVA, S. "CNC-Programação de comandos numéricos computadorizados torneamento". Ed. Érica Ltda, São Paulo, 2002.

SHOPCNC. A Indústria dos Robôs. 2020. Disponível em: https://www.shopcnc.com.br/produtos/manutencao-de-robos-abb/manutencao-de-robos. Acesso em: 23 jan. 2021. 


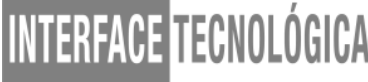

SOUZA, C. E. D. de. Vantagens e Desvantagens do Controle Numérico

Computadorizado (CNC). 2020. Disponível em:

https://www.google.com/search?q=20.pdf\&oq=20.pdf\&aqs=chrome..69i57\&sourceid=chrom e\&ie=UTF-8. Acesso em: 20 jan. 2021.

TESSARINI JUNIOR, G.; SALTORATO, P. Impactos da Indústria 4.0 na organização do trabalho: uma revisão sistemática da literatura. Revista Produção Online. Florianópolis, SC, v. 18, n. 2, p. 743-769, 2018. Disponível em: producaoonline.org. Acesso em: 10 jan. 2021. 